Schleiermachers Hermeneutik

und ihre Vorgeschichte im 18. Jahrhundert 


\author{
Harald Schnur
}

\title{
SCHLEIERMACHERS HERMENEUTIK UND IHRE VORGESCHICHTE IM 18. JAHRHUNDERT
}

Studien zur Bibelauslegung, zu Hamann, Herder und F. Schlegel

Verlag J. B. Metzler Stuttgart - Weimar 
Die Deutsche Bibliothek - CIP-Einheitsaufnahme

Schnur, Harald:

Schleiermachers Hermeneutik und ihre Vorgeschichte im 18. Jahrhundert:

Studien zur Bibelauslegung, zu Hamann, Herder und F. Schlegel / Harald Schnur. -

Stuttgart; Weimar: Metzler, 1994

ISBN 978-3-476-00995-1

ISBN 978-3-476-00995-1

ISBN 978-3-476-03529-5 (eBook)

DOI 10.1007/978-3-476-03529-5

Dieses Werk einschließlich aller seiner Teile ist urheberrechtlich geschützt. Jede Verwertung außerhalb der engen Grenzen des Urheberrechtsgesetzes ist ohne Zustimmung des Verlages unzulässig und strafbar. Das gilt insbesondere für die Vervielfältigungen, Übersetzungen, Mikroverfilmungen und die Einspeicherung und Verarbeitung in elektronischen Systemen.

(C) 1994 Springer-Verlag GmbH Deutschland

Ursprünglich erschienen bei J.B.Metzlersche Verlagsbuchhandlung und Carl Ernst Poeschel Verlag GmbH in Stuttgart 1994 


\section{Inhalt}

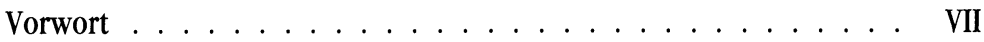

1. Einleitung - Friedrich D. E. Schleiermacher: "Zur Nothwendigkeit einer allgemeinen Hermeneutik"

1.1. Schleiermachers Zäsur in der Geschichte der Hermeneutik . . . 1

1.2. Ein Ursprung in kategorialer Nichtverständlichkeit? . . . . . . 4

1.3. Gadamer und Dilthey über die Vorgeschichte . . . . . . . . 5

1.4. Darstellungen anderer . . . . . . . . . . . . . . . . 11

1.5. Aufriß der Untersuchung . . . . . . . . . . . . . . . . . . . . 19

1.6. Zum aktuellen Stand der Forschung . . . . . . . . . . . 21

2. Die Tradition der Bibelhermeneutik: "Jedermann ist seiner eigenen Worte [...] einziger Ausleger"

2.1. Einleitung . . . . . . . . . . . . . . . . . . 27

2.2. Die Entflechtung von Hermeneutik und Dogmatik _ . . . . 28

2.3. Johann August Ernesti: "Institutio Interpretis

Novi Testamenti« (1761) . . . . . . . . . . . . . . . . 34

2.4. Die Historisierung der Bibelauslegung . . . . . . . . . . . 37

2.5. Die grammatisch-historische Interpretation . . . . . . . . 46

2.6. Das Motiv der Bibelhermeneutik . . . . . . . . . . . . . 51

3. Johann Georg Hamann: »Philolog ärgert sich über den exegetischen Materialismum"

3.1. Einleitung . . . . . . . . . . . . . . 59

3.2. Hamanns Theologie: Kondeszendenz Gottes _ . . . . . . 62

3.3. Geist und Buchstabe . . . . . . . . . . . . . . . . . . . 68

3.4. Hamann über die Sprache $\ldots \ldots \ldots$. . . . . . . . . . . 77

3.5. Hermeneutische Argumentationen . . . . . . . . . 84

4. Johann Gottfried Herder: „bei mir lebte, da ich las"

4.1. Einleitung . . . . . . . . . . . . . . . . . . . 97

4.2. Die Absetzung von Hamann $\ldots \ldots$

4.3. Gnoseologie und Gefühl . . . . . . . . . . . . . . . . . 102

4.4. Menschliche Bibelauslegung . . . . . . . . . . . . 106

4.5. Geschichtsphilosophie des Individuellen . . . . . . . . . . 115

4.6. Analogisierendes Verstehen . . . . . . . . . . . . . . . 122

4.7. Sprache und Literatur . . . . . . . . . . . . . . . . . . . 124

4.8. $\mathrm{Kraft} \ldots \ldots \ldots \ldots \ldots \ldots \ldots$ 


\section{Inhalt}

5. Friedrich Schlegel: »Die Lectüre ist eine Kunst»

5.1. Einleitung. . . . . . . . . . . . . . . . . . . 139

5.2. Zu Schlegels frühromantischer Periode . . . . . . . . . . . . . 140

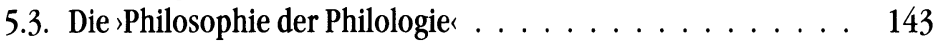

5.4. Zyklisches Lesen . . . . . . . . . . . . . . . . . . . . . 145

5.5. Verstehen, Unverständlichkeit, Besserverstehen . . . . . . . 147

5.6. Schlegel und die Hermeneutik Schleiermachers . . . . . . . . . 152

6. Schleiermachers Hermeneutik und ihre Vorgeschichte: "die Praxis wird [...] mit der Theorie eine bewußtere"

6.1. Einleitung ....................... 161

6.2. Das System des Wissens . . . . . . . . . . . . . . . . . . . 162

6.3. Denken, Sprechen, Verstehen . . . . . . . . . . . . . 168

6.4. Die Gestalt der Hermeneutik . . . . . . . . . . . . . . . . . . 170

6.5. Zusammenfassung: Verhältnisbestimmung zur Vorgeschichte . . . . . . . . . . . . . . . . 179

Literaturverzeichnis

A. Quellen

A.a. Bibelhermeneutik . . . . . . . . . . . . . . 193

A.b. Andere . . . . . . . . . . . . . . . 196

B. Forschungsbeiträge ................... 201 


\section{Vorwort}

Jede Untersuchung tritt mit dem Anspruch auf, etwas Neues zutage gefördert zu haben. Das ist auch hier nicht anders. Allerdings wird dieser Anspruch in einer ungewöhnlichen Situation geltend gemacht.

Das Thema der vorliegenden Untersuchung gilt nämlich in der Forschung als abschließend bearbeitet. Die Akten scheinen - um eine bildliche Ausdrucksweise zu wählen - längst geschlossen zu sein, weil das Urteil gefällt, angenommen und ein Revisionsantrag nicht zu erwarten ist. Den stellt aber diese Untersuchung, welche die Akten auch neu öffnet, die Urteilsfindung neu durchführt und zu einem neuen, deutlich anderslautenden Urteil findet.

Zuvor aber ist die Notwendigkeit zu begründen, das Procedere zu wiederholen - wenn wir darin einig sind, daß am Ende ein Urteil der Sache angemessen sein soll. Dies geschieht, indem die Einleitung in die vorliegende Untersuchung für das in Kraft befindliche Urteil nachweist, daß es durch Verfahrensfehler zustande gekommen ist. Angesichts der Bedeutung des Themas und der - wie sich herausstellen wird - zu Unrecht verbreiteten Sicherheit hinsichtlich seiner Einschätzung hat die Einleitung den Status eines eigenen Kapitels angenommen. Auch das ist ungewöhnlich und verdient eine Erläuterung. Mit dem einleitenden Kapitel 1 sollen die Gründe für die Verfahrensfehler deutlich werden. Dabei wird einsichtig, daß es der Einfluß von Wilhelm Dilthey und Hans-Georg Gadamer war, der eine (insgesamt gesehen) unangemessene Sicht der Dinge hervorgerufen und befestigt hat.

Im Gegenzug entwirft Kapitel 1 einen Untersuchungsrahmen, der ein begründetes Urteil ermöglicht. Dieses Feld ist, um auch dazu gleich eine Erläuterung zu geben, disziplinenübergreifend konzipiert. Ich war mir in jeder Arbeitsphase darüber im klaren, daß es unmöglich ist, den Ansprüchen der jeweiligen, hier einschlägigen Fächern gerecht zu werden: Die Theologie dürfte eine stärkere Durchdringung der theologiegeschichtlichen Umwälzungen vom 18. zum 19. Jahrhundert (Aufklärung bis Schleiermacher) anmahnen, die Germanistik hinsichtlich der Literatur (Aufklärung bis Sturm und Drang, Klassik und Romantik) und die Philosophie mit gleichem Recht dasselbe für ihren Bereich (Aufklärung bis Kant und die Folgen). Die (Alt-) Philologie könnte ihre Geschichte überhaupt zu wenig berücksichtigt sehen. Allerdings wird hier eben auch ein Thema bearbeitet, das Anteile aus allen diesen Disziplinen in sich vereinigt und deshalb nicht mit einer von ihnen zusammenfällt. Es ist gerade ein Ergebnis der kritischen Durchsicht vorliegender Arbeiten zu diesem Bereich, wie sie im einleitenden Kapitel geschehen wird, daß diese in der einen oder anderen Form einen disziplinär verengten Blick eingenommen haben. Die Konzeption der Untersuchung soll insofern als Korrektiv für die allzu eingegrenzte fachliche Beurteilung dienen. 
Ihr Text stellt eine überarbeitete Fassung meiner Konstanzer Dissertation dar. Sie konnte unter sehr günstigen Arbeitsbedingungen entstehen. Auch dafür, neben so vielem, habe ich meinem Betreuer, Prof. Dr. Jürgen Mittelstraß, sehr zu danken. Sollte das Verständnis von Wissenschaft als Lebensform aus dem Blickwinkel eines Doktoranden heißen, ihm (geduldig) so viel Freiheit wie möglich und (weniger geduldig) so viel Führung wie nötig zu geben, dann habe ich das über einige Jahre erfahren können. Von großem Vorteil hat sich erwiesen, mit Prof. Dr. Gerhart von Graevenitz einen Germanisten zum zweiten Gutachter wie Betreuer gewonnen zu haben. Ich danke ihm ebenfalls sehr: Die Kompetenzen haben sich aufs beste ergänzt; das Projekt hat sehr davon profitiert.

Die Studienstiftung des deutschen Volkes gewährte für das Vorhaben ein Promotionsstipendium. Für diese finanzielle Grundlage möchte ich meinen Dank aussprechen.

Schließlich habe ich meinen Eltern, Heinrich und Erika Schnur, für ihre moralische und monetäre Unterstützung (das eine kontinuierlich, das andere okkasionell) herzlich zu danken, insbesondere für ihre Geduld mit einem ihnen so wenig vertrauten Verfahren.

Einige technische Bemerkungen: Die Belegstellen werden mit Autorname und Erscheinungsjahr sowie gegebenenfalls Bandzahl angeführt und sind über das Literaturverzeichnis zu vervollständigen. Siglen tragen nur Quellentexte; Hervorhebungen sind generell nicht übernommen. Zitate ohne Anmerkung zählen zur nächstfolgenden; werden sie mit einem Punkt geschlossen, muß dieser nicht in jedem Fall mit einem Satzende an der originalen Stelle übereinstimmen. Enthalten sie runde Klammern, so wurden Textergänzungen von Herausgebern über- oder Wortumstellungen vorgenommen; eckige Klammern dagegen stammen von mir. Wegen der disziplinenübergreifenden Anlage der Untersuchung ist das (aktualisierte) Verzeichnis der Forschungsbeiträge alphabetisch geordnet. Die Untersuchung ist konzeptionell nicht mit editorisch-philologischen Fragen befaßt; wo es möglich war, hat sie aber stillschweigend einen als zuverlässig geltenden Text zur Referenz gewählt. 